\title{
IMPACTO DEL PLAN COMUNAL UNIVERSITARIO EN EL FORTALECIMIENTO DEL SISTEMA DE EXTENSIÓN Y PROMOTORÍA RURAL EN NICARAGUA
}

\section{IMPACT OF THE UNIVERSITY COMMUNITY PLAN IN THE STRENGTHENING OF THE EXTENSION AND RURAL PROMOTORY SYSTEM IN NICARAGUA}

\section{Aguilar-Bustamante Víctor ${ }^{1}$, Nicaragua Karla ${ }^{2}$}

${ }^{1}$ Universidad Nacional Agraria, Managua, Nicaragua, e-mail: victor.aguilar@una.edu.ni

${ }^{2}$ Instituto Nicaraguense de Tecnología Agropecuaria (INTA)

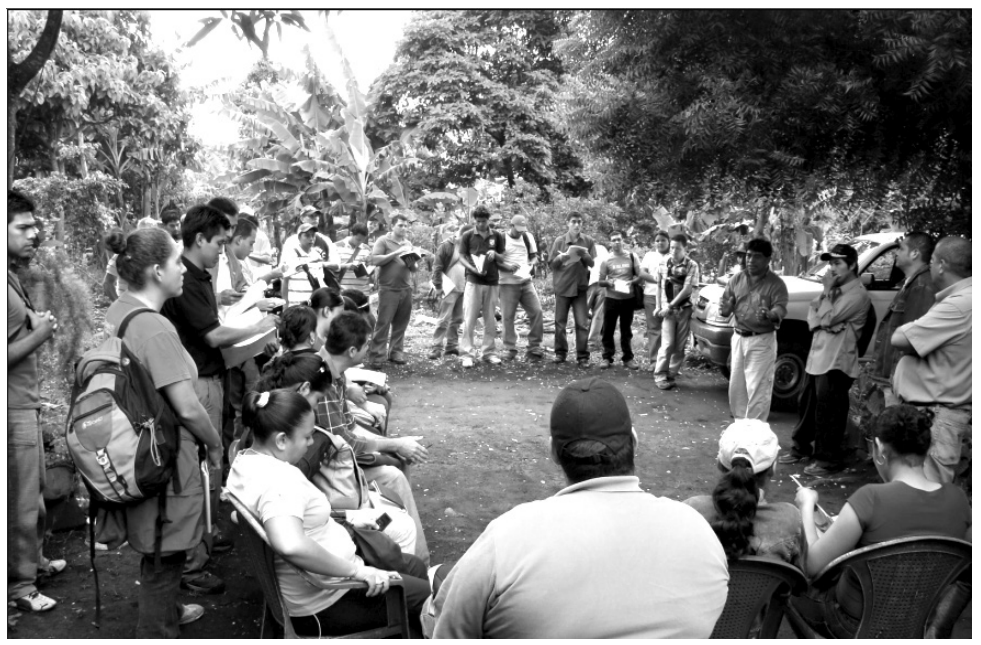

\section{RESUMEN}

El Plan Comunal Universitario (PCU) fue iniciado de Mayo a Diciembre del año de 2009 entre las universidades del Consejo Nacional de Universidades (CNU) y la Oficina de Desarrollo Institucional (ODI) del Instituto Nicaragüense de Tecnología Agropecuaria (INTA). El financiamiento fue obtenido del Fondo para el Desarrollo de Capacidades del PRORURAL (FADECAPRO). Se involucraron un total de 136 egresados y graduados de las carreras de Agronomía, Veterinaria, Zootecnia, Ingeniería Agrícola, Economía, comunicación, Ingeniería en Sistemas y Antropología de la Universidad Nacional Agraria (UNA), Universidad Nacional de Ingeniería (UNI), Universidad Nacional Autónoma de Nicaragua Managua (UNAN Managua), Universidad Nacional Autónoma de Nicaragua de León (UNAN León), Universidad Católica del Trópico Seco (UCATSE), Escuela internacional de Agricultura y Ganadería de Rivas (EIAG), Universidad Centroamerciana (UCA), Bluefield Indian and Caribbean Universitiy (BICU) y la Universidad de las Regiones Autónomas de la Costa Caribe Nicaragüense (URACCAN). El curso de inducción, el curso de promotoría rural y demás capacitaciones fueron impartidas en el CNIAB - INTA Managua, Campos Azules INTA Masatepe, INTA Juigalpa, Centro del Valle de Sébaco -CEVAS INTA Matagalpa y en el Centro Experimental de Occidente -CEO León. Los objetivos del PCU fueron los de capacitar a recién egresados y graduados de las Universidades, fortalecer la red de promotores rurales, aumentar la cobertura de asistencia técnica del INTA y mejorar la seguridad alimentaria y nutricional de las familias campesinas. Se logró organizar a un total de 588 promotores y beneficiar a través del proceso de capacitación y transferencia tecnológica a un total de 10571 productores de los cuáles $42 \%$ fueron mujeres. Se logró

\section{ABSTRACT}

The University Community plan (PCU) started from May to December 2009 between the National University Counsel (CNU) and the Institutional Development Office (ODI) from the Nicaraguan Institute of Agrarian technology (INTA). The financial support was taken from the capacity development fund of PRORURAL (FADECAPRO). To the project were integrated 136 new graduated student of the following careers of veterinary medicine, animal husbandry, economist, communication, computer systems and anthropology of National Agrarian University (UNA), Engineer National University (UNI), Autonomous National University of Nicaragua (UNAN Managua and UNAN León), Catholic University of dry tropic (UCATSE), Rivas Agriculture International school (EIAG), Central American University (UCA), Bluefield Indian and Caribbean University (BICU) and the University of Autonomous Regions of Caribbean Cost of Nicaragua (URACCAN). The starting course, the rural promontory course and remainder technical training were given in CNIAB INTA Managua, Campos Azules INTA Masatepe, INTA Juigalpa, CEVAS INTA Matagalpa and CEO INTA León. The objectives of the PCU were strengthening the professional capacities, strengthening the net of rural promoters, increase the INTA technical assistance in the production areas and improve the food security and nutrition quality of poorer families of the rural sector. At the end of December 2009, a total of 588 rural promoters were organized and trained and a total of 10571 producers were benefited with INTA technical assistance where $42 \%$ were women. The cover of INTA technical assistance, were increased in $12 \%$. Those new professionals with their work let an unforgettable footprint in the communities and cooperatives and also and their own life. With this kind of agreement between 
incrementar en un $12 \%$ el número de beneficiarios o cobertura del INTA con los servicios de asistencia técnica pública. La vitalidad de estos jóvenes, protagonizó una faena técnica y humana y cuyas repercusiones dejaron una profunda huella en las comunidades y cooperativas que ellos atendieron y una huella en sus propias vidas, lo cual se resalta en un incremento de la autoestima y la buena voluntad de un relevo generacional responsable y comprometido con el desarrollo de Nicaragua.

Palabras clave: Plan Comunal Universitario, CNU, INTA, FADECAPRO, Promotoría Rural

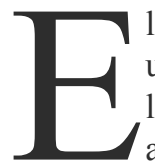
1 Plan Comunal Universitario (PCU), nace como una propuesta del gobierno para involucrar a todos los recién egresados y graduados de las carreras afines al agro, al quehacer de las instituciones del gobierno como el Instituto Nicaragüense de Tecnología Agropecuaria (INTA). Como es conocido, el INTA por sí solo, año con año no puede ofrecer asistencia técnica a más de 300000 pequeños y medianos productores de Nicaragua; así surge la idea de involucrar a los nuevos profesionales egresados de las diferentes universidades, en asistencia técnica directa, asistencia técnica a cooperativas, elaboración de proyectos para los Centros de Desarrollo Tecnológico (CDT) del INTA, mantenimiento y actualización de los equipos de información, mantenimiento e inventario de toda la maquinaria agrícola y de riego existente en cada uno de los CDT y fortalecer la red de los promotores rurales creada por el INTA a nivel nacional.

Es bien conocido que el sector agropecuario aporta aproximadamente el $20 \%$ del Producto Interno Bruto (PIB) a nivel nacional y aporta un $27 \%$ del empleo productivo total lo que hace ser un sector muy relevante para la economía nacional (BCN, 2010). Aún sabiendo que los medianos y pequeños productores controlan el $70 \%$ de la tierra y que aportan un $65 \%$ de los alimentos que consume la población nicaragüense, también es muy conocido que un porcentaje bastante alto de un $67.8 \%$ de la población vive en pobreza (FIDEG, 2009).

El sector productivo enfrenta grandes desafíos como son incrementar la productividad ya que la producción se ha basado en la expansión de áreas y bajo nivel tecnológico y aunado a esto el uso de tierras marginales para la agricultura y los efectos del cambio climático. El poco valor agregado a los productos vuelve más vulnerable al sector productivo ya que sus ganancias se ven más reducidas (FUNICA, 2011). Es por eso que el INTA se ha planteado con la integración de los nuevos profesionales aumentar su cobertura llevando a los productores las nuevas innovaciones tecnológicas, transfiriendo nuevos conocimientos a través de la prospección de nuevas tecnologías para elevar la productividad y mejorar el nivel de vida de las familias rurales pobres.

Este modelo de Promotoría rural, surge como necesidad del INTA de capacitar a capacitadores en los CDT, en las parcelas de los promotores rurales y de pequeños y medianos productores. Fortalecer las capacidades locales
$\mathrm{CNU}$ and the public institutions of the government we are preparing the new generation of young professionals to contribute to the Nicaraguan development.

Keywords: University Community Plan, CNU, INTA, FADECAPRO, Rural Promotory.

de las familias productoras y así contribuir al desarrollo productivo, socio-económico y ambiental, mediante procesos de enseñanza aprendizaje que les permita aplicar correctamente tecnologías en respuesta a sus necesidades y oportunidades (INTA, 2009).

La promotoría rural es un proceso educativo no formal basado en la enseñanza y aprendizaje de productor a productor con la ayuda del extensionista. Es un proceso de transferencia de conocimientos, habilidades y valores a otras familias productoras, tomando en cuenta el principio andragógico de educación de adultos "Aprender-Haciendo". Está basado en el liderazgo local, en cambios de actitud para que adopten las técnicas y tecnologías necesarias mejorando así su productividad, ingresos y el bienestar personal, familiar y comunitario.

El Plan Comunal Universitario en su fase inicial fue llevado a cabo de Mayo a Diciembre de 2009 con un total de 136 profesionales recién egresados donde 71 egresados se dedicaron a la promotoría rural, 22 se ubicaron en los Centros de Desarrollo Tecnológico y 43 en otras tareas propias del INTA. Los objetivos del PCU fueron los de capacitar a egresados y graduados de las Universidades del CNU, fortalecer la red de promotores rurales, aumentar la cobertura de asistencia técnica del INTA y mejorar la seguridad alimentaria y nutricional de las familias campesinas.

\section{METODOLOGÍA}

En febrero de 2009, se convocó a los rectores de las 10 universidades miembros del CNU para explicar el PCU y que cada una de las universidades designara un representante para la convocatoria, organización y seguimiento del PCU 2009. La meta planteada era involucrar a 150 nuevos profesionales distribuidos 100 en promotoría rural y 50 en otras actividades del INTA. La meta planteada era que cada uno de los nuevos profesionales que se capacitara como promotor rural capacitara a 10 promotores rurales de las comunidades para alcanzar un total de 1000 promotores rurales capacitados de acuerdo a las necesidades de los productores (currícula) de cada comunidad y que cada promotor rural de cada comunidad diera asistencia técnica a 10 productores y lograr que 10000 productores recibieran asistencia técnica.

Para el PCU 2009, una vez conocida la demanda de los productores, de las cooperativas y de los CDT del INTA, se convocó a los egresados y graduados de los años 2007 y 
2008. El tiempo de participación sería de entre 6 y 12 meses como asistente técnico o promotor rural. De preferencia el participante debería quedar en el lugar de origen ya que no había suficientes fondos para cubrir todos los gastos. En la figura 1 se puede ver la procedencia de los nuevos egresados y graduados de las diferentes universidades que participaron en el PCU 2009.

Los fondos para el Plan Comunal Universitario fueron tomados del Fondo para el Desarrollo de Capacidades del PRORURAL -FADECAPRO y cubrieron el estipendio mensual de los participantes, el seguimiento al plan comunal por parte de INTA, CNU e IICA y el apoyo para la realización de los diferentes talleres para la promotoría rural y capacitaciones. Cada uno de los egresados y graduados recibió un estipendio mensual de 3000 córdobas netos para su alimentación y transporte, además de materiales didácticos como mochila, botas, calculadoras, capotes, camisetas, libretas y lapiceros. Se firmaron acuerdos para que las cooperativas apoyaran con alojamiento y alimentación a los participantes según su ubicación.

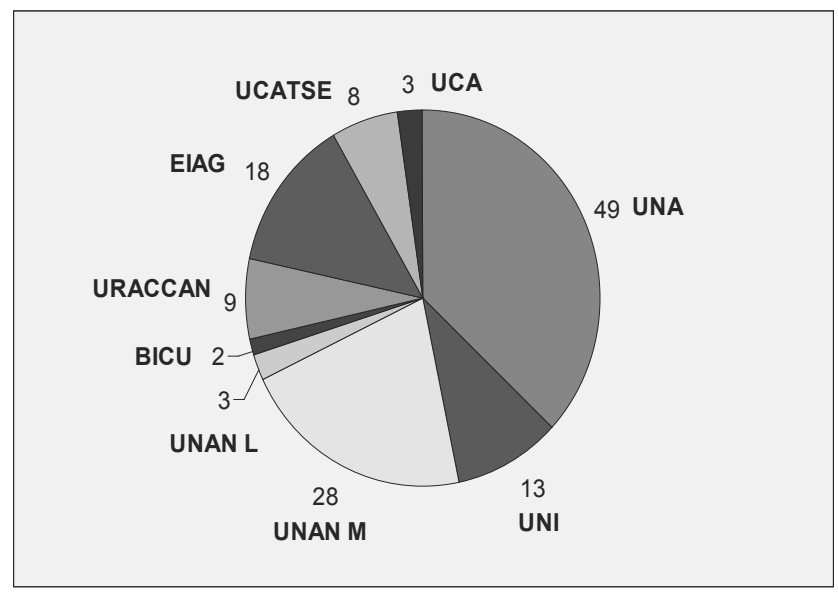

Figura 1. Procedencia de los nuevos profesionales ubicados en las cooperativas y Centros de Desarrollo Tecnológico del INTA 2009.

El PCU fue voluntario pero se impregnó en ellos una gran responsabilidad para sí mismo, para la universidad del cuál egresaron, para el INTA y sobre todo para los productores que esperaban de los integrantes del PCU un giro significativo en los diferentes sistemas de producción. La formalización de los participantes en el PCU se hizo a través de una carta compromiso entre el INTA y el participante, así mismo se llenó una ficha de registro de los principales datos del egresado.

De acuerdo a la demanda recibida por parte de INTA, se distribuyeron los nuevos profesionales según el lugar de procedencia o en último caso donde el participante desease ejercer su trabajo. En la figura 2, se puede observar la ubicación de los participantes en cada una de las regiones. La demanda estuvo distribuida de la siguiente manera: 9 en el INTA Central, 11 en los diferentes distritos de Managua, 17 en el CNIAB, 34 en la zona pacífico sur (Masaya, Granada,
Carazo y Rivas), 25 en el Centro Sur (Juigalpa, Boaco, Rio San Juan, Nueva Guinea, Rama y Bluefield), 5 en Ziuna, 13 en el centro Norte (Matagalpa y Jinotega), 9 en Las Segovias (Estelí, Madriz y Nueva Segovia) y 14 en Pacífico norte (León y Chinandega).

El PCU inició con un curso de inducción y fue realizado en el CNIAB, Campos Azules (CECA) INTA Masatepe, INTA Juigalpa, Centro Experimental del Valle de Sébaco (CEVAS) INTA Matagalpa y en el Centro Experimental de Occidente (CEO) INTA León. Los integrantes del PCU ubicados en Ziuna fueron capacitados en el CEVAS INTA Matagalpa. En el curso de inducción se dio a conocer el esquema organizativo del INTA para la apropiación del mismo. Además se explicaron las responsabilidades que adquiría el participante al inscribirse e iniciar con el curso de inducción, ya que aunque fuese voluntario no podrían renunciar cuando ellos quisieran, además todos los participantes se someterían a los lineamientos y políticas del INTA y deberían de cumplir con un horario tal como si fuesen trabajadores permanentes del INTA.

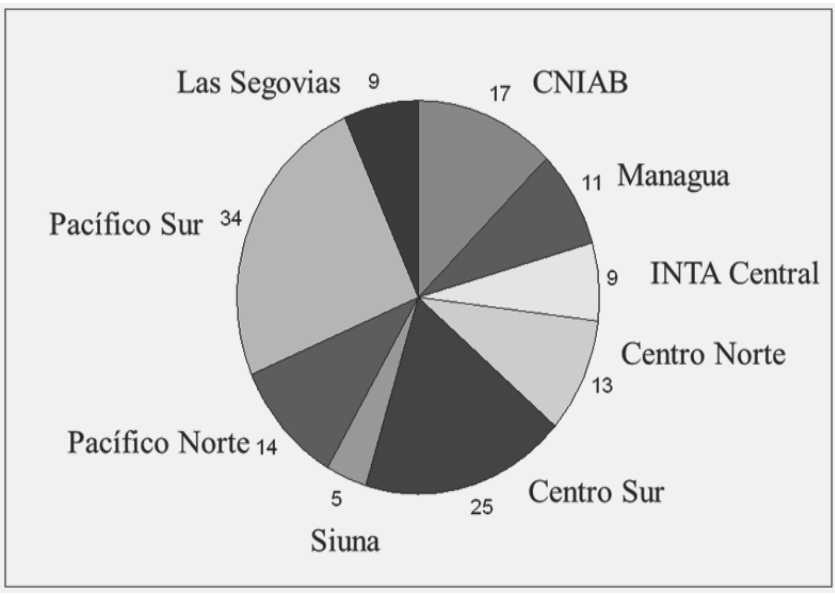

Figura 2. Ubicación de los nuevos profesionales en las cooperativas y centros de Desarrollo tecnológico de los diferentes departamentos de Nicaragua.

El segundo paso fue la realización del taller de promotoría rural que estuvo a cargo de especialistas en promotoria rural del INTA. Cada uno de estos talleres fue realizado en los todos los centros antes mencionados. Se compartieron experiencias entre los nuevos profesionales o nuevos promotores rurales y promotores rurales ya organizados por el INTA y que contaban con mucha experiencia en capacitación a los productores de las comunidades cercanas a su domicilio (Ver foto 1$)$.

La tercera etapa fue la capacitación técnica de los participantes del PCU a lo largo del segundo semestre de acuerdo a la una currícula de acuerdo a la zona y necesidades de las comunidades donde estarían ubicados los egresados y graduados. Ver tabla 1.

Antes de iniciar con las capacitaciones en cada uno de los lugares, los participantes del PCU realizaron un 
diagnóstico para conocer las necesidades de los productores de la comunidad y se procedió a diseñar una currícula para capacitar a los 10 promotores rurales seleccionados desde mayo a diciembre de 2009.

Posteriormente, con la ayuda de los líderes comunales como los Consejos del Poder Ciudadano, líderes de las cooperativas, organizaciones comunales y gobiernos locales, se eligieron a los 10 promotores rurales que serían capacitados según la demanda de la comunidad. En las comunidades donde había promotores rurales se procedió a ratificarlos en común acuerdo con la población. A medida que avanzó el PCU se fue impartiendo el módulo de capacitación técnica según la ubicación de los participantes.

Tabla 1. Talleres de capacitación para los egresados y graduados del PCU 2009

\begin{tabular}{|c|c|c|c|}
\hline Zona & Capacitaciones & Participantes & Temática de los talleres \\
\hline C Norte & 5 & 13 & $\begin{array}{l}\text { Inducción, Promotoría Rural } \\
\text { Hortalizas, semillas y género. }\end{array}$ \\
\hline C Sur & 5 & 25 & $\begin{array}{l}\text { Inducción, Diagnóstico Participativo Comunitario } \\
\text { (DPC), Promotoría, asociativismo, hortalizas, nutrición } \\
\text { bovina, alimento de verano, cacao y post cosecha. }\end{array}$ \\
\hline P Norte & 2 & 14 & $\begin{array}{l}\text { Inducción, promotoría, manejo silvopastoril, BPA y } \\
\text { establecimiento de sistemas agroforestales. }\end{array}$ \\
\hline P Sur & 10 & 34 & $\begin{array}{l}\text { Inducción, promotoría, DPC, comunicación, planes de } \\
\text { negocios, técnicas MIC en granos básicos, hortalizas, } \\
\text { nutrición bovina, aves y cerdos. }\end{array}$ \\
\hline L Segovias & 3 & 8 & $\begin{array}{l}\text { Inducción, promotoría, métodos de extensión y manejo } \\
\text { de SPSS }\end{array}$ \\
\hline Managua & 1 & 11 & $\begin{array}{l}\text { Inducción, promotoría, mapeos, diagnóstico de } \\
\text { necesidades para capacitación. Extensión agropecuaria, } \\
\text { producción de semillas de granos básicos, hortalizas, } \\
\text { manejo zoosanitario y nutrición bovina. }\end{array}$ \\
\hline Siuna & 2 & 5 & Inducción y promotoría rural \\
\hline Total & 28 & 110 & \\
\hline
\end{tabular}

Los criterios para seleccionar a los promotores rurales fueron los de ser productor agropecuario, manejar adecuadamente su finca, usar su finca para transferencia tecnológica, residir en la comunidad, saber leer y escribir, ser líder, ser electo por la comunidad, ser colaborador, si disponía de tiempo, que estubiera dispuesto a capacitarse y que estubiera dispuesto a transmitir los conocimientos a los demás productores (INTA, 2010).

\section{RESULTADOS}

El PCU terminó en diciembre 2009 con 136 participantes. De 100 participantes como promotor rural que estaba previsto solo se involucraron 71 para un cumplimiento de un $71 \%$ lo que significa $52 \%$ del total de los 136 participantes. De los 71 promotores 34 se ubicaron en cooperativas y 37 en las comunidades. Se ubicaron 18 en Centro Sur, 5 en Siuna, 6 en Pacífico Norte, 7 en Centro Norte, 20 en Pacífico Sur, 9 en Managua y 6 en Las Segovias. Del total de los 136 participantes $22(16 \%)$ fueron ubicados en los CDT del INTA y 43 (32\%) en áreas de administrativas de la misma institución. De los 22 egresados que fueron ubicados en los CDT del INTA, 9 fueron ubicados en el CNIAB desempeñándose en áreas de producción de semilla certificada y en las áreas de producción agrícola donde realizaron labores muy importantes como actualización del inventario de semillas, inventario de maquinaria agrícola y riego, elaboración de una propuesta política ambiental interna e identificación de legislación ambiental aplicable para el CNIAB, mayor atención a cultivos en áreas experimentales y comerciales. Por parte del INTA se involucraron 28 técnicos que son trabajadores permanentes con mucha experiencia y que acompañaron a los nuevos egresados y graduados en la primera fase.

El PCU terminó el año 2009 con una capacitación promedio de 8 promotores rurales de los 10 planificados. En la zona de Centro Sur se presentó la relación más baja de 3 promotores por participante debido al reducido número de cooperativas, En Ziuna y pacífico sur se presentó una relación promedio de 7 promotores rurales por egresado o graduado. Al contrario en Las Segovias y en Centro Norte se capacitó a un promedio de 10 promotores rurales por participante del PCU logrando en este caso la meta prevista, en Managua se capacitó a un promedio de 11 y en pacífico norte un promedio de 12 promotores por participante.

De igual manera se logró atender a 8 productores por cada productor capacitado o promotor rural capacitado por los participantes del PCU. Las metas de capacitar a 1000 promotores rurales no se lograron debido a que no se pudo completar el número de 150 egresados y graduados solicitado por INTA, por lo que se procedió a ubicar solamente a 71. El PCU estaba iniciando y los presidentes de las cooperativas no estaban claros de que profesional solicitar y que fuese útil en las labores de producción de los productores de las cooperativas retrasando un poco la distribución de los participantes del PCU. No se logró contactar la totalidad de los productores independientes y a los presidentes de las cooperativas de cada zona por falta de logística y capacidad del personal de los zonales del INA.

En total de organizaron y capacitaron 588 promotores rurales cuya distribución se puede observar en la figura 3 . En Managua se organizaron y capacitaron 100 promotores rurales, en pacífico norte 69 , en pacífico sur 199 , en centro 
sur 54, centro norte 67, en Siuna 37 y en las Segovias 62 promotores rurales o productores rurales.

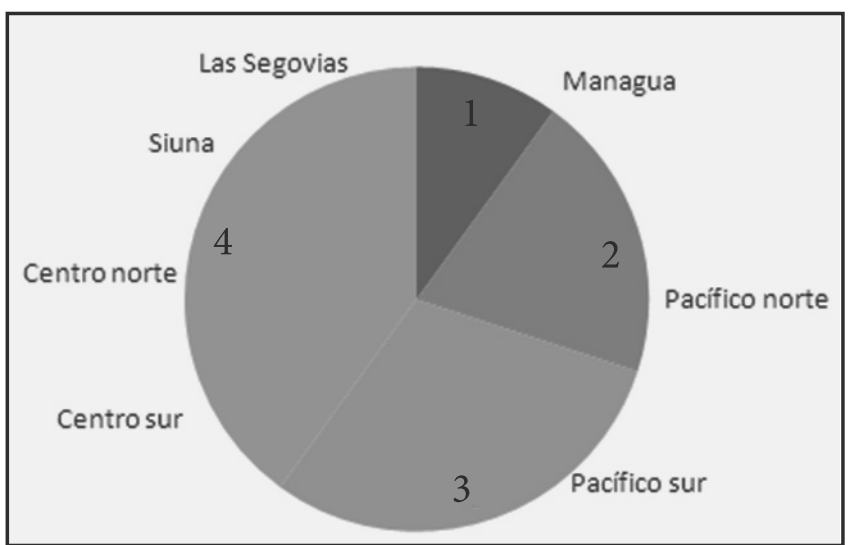

Figura 3. Distribución de la organización y capacitación de los promotores rurales por parte de los integrantes del PCU y técnicos permanentes del INTA, 2009.

Los egresados y graduados se incorporaron en cuatro niveles de trabajo

A nivel de cooperativas. Un total de 34 egresados se ubicaron en 25 cooperativas en todo el país, como en Siuna 5 cooperativas, Centro Sur 8 cooperativas, Centro Norte 7 cooperativas, Pacífico Norte 2 cooperativas y en Las Segovias 3 cooperativas.

A nivel de comunidades. En las zonas de Pacífico Sur y Managua que por sus características no se logró insertar a los egresados en cooperativas, se ubicaron en comunidades para ejercer la promotoría rural. A nivel de las comunidades se insertaron 37 egresados y graduados. Estos dieron seguimiento a los procesos iniciados por los técnicos del INTA o técnicos que se retiraron del territorio.
A nivel de Centros de Desarrollo Tecnológico. De los 136 participantes 22 se ubicaron en los CDT del INTA. Las tareas desarrolladas fueron mantenimiento constante y regulación de la maquinaria agrícola, preparación adecuada y oportuna de la tierra para la siembra, prevención de plagas de los cultivos, riego apropiado a las áreas de siembra, mejor distribución de la carga de trabajo en las áreas productivas, lográndose cumplir tareas en el tiempo planificado, ordenamiento de las áreas de siembra basado en un análisis de las características de suelo del CNIAB, realización de capacitaciones a obreros agrícolas sobre la colocación de residuos y actualización de inventarios de maquinaria agrícola y de riego.

A nivel de oficinas del INTA Central y Zonal. Se insertaron un total de 43 egresados y graduados, 17 en el INTA central, 11 en las oficinas de Pacífico Sur, Oficinas zonales Pacífico Norte (4), Centro Sur (4), Centro Norte (3), Las Segovias (2) y zonal Managua (2). Las actividades realizadas fueron fortalecer el área de proyectos, sistematizaciones de las Escuelas de Campo -ECA, Promotoría y adopción de sistemas agroforestales. Actualización de la contabilidad del Centro Experimental de Campos Azules -CECA en Masatepe, desarrollo en capacidades en género, realización de estudios de caso, mayor agilidad y calidad en el servicio de asistencia técnica en informática y ordenamiento y procesamiento de información de diagnósticos agrosocioeconómicos.

De mayo a diciembre de 2009, el PCU logró la ejecución de actividades en todo el territorio nacional incorporando al proceso de asistencia técnica a un total de 10571 productores rurales entre promotores rurales y productores (tabla 2). Del total de los beneficiados 4394 fueron mujeres para un $42 \%$ de participación.

Tabla 2. Número de promotores rurales y productores atendidos por 71 participantes del Plan Comunal Universitario y 28 técnicos del INTA. Nicaragua 2009

\begin{tabular}{lccc}
\hline & Numero de promotores rurales y productores atendidos \\
\hline Zona & Hombres & Mujeres & Total \\
\hline Siuna & 244 & 136 & 380 \\
Pacífico Sur & 541 & 207 & 748 \\
Pacífico norte & 53 & 60 & 113 \\
Centro sur & 3815 & 2918 & 6733 \\
Centro norte & 694 & 283 & 977 \\
Las Segovias & 315 & 305 & 620 \\
Managua & 515 & 485 & 1000 \\
& & & \\
\hline Total & 6177 & 4394 & 10571 \\
\hline
\end{tabular}




\section{REFERENCIAS BIBLIOGRÁFICAS}

FIDEG (Fundación Internacional para el Desafío Económico Global). 2009. Encuesta de hogares para la medición de la pobreza en Nicaragua. 30p.

FUNICA (Fundación para el Desarrollo Tecnológico, Agropecuario y Forestal de Nicaragua). 2011. La importancia de la prospección tecnológica en el sector agropecuario de Nicaragua. Boletín Informativo Diciembre 2011.

INTA (Instituto Nicaragüense de Tecnología Agropecuaria). 2009. Proyecto de fortalecimiento del sistema de extensión rural con énfasis en la zona Centro Sur para mejorar la seguridad alimentaria y nutricional de las familias campesinas. Informe Final. 42p. INTA, diciembre 2009 reporte de finalización del PCU - CNU - INTA 2009.

INTA (Instituto Nicaragüense de Tecnología Agropecuaria). 2010. Guía del promotor y promotora rural agropecuario. Edición 5. Mayo 2010. 